\title{
Tooth loss patterns in older adults with special needs: a Minnesota cohort
}

\author{
Xi Chen*, Jennifer J. Clark \\ Department of Dental Ecology, University of North Carolina, Chapel Hill, NC 27599, USA
}

This study was conducted to detail tooth loss patterns in older adults with special needs. A total of 491 elderly subjects with special needs were retrospectively selected and followed during 10/1999-12/2006. Medical, dental, cognitive, and functional assessments were abstracted from dental records and used to predict risk of tooth loss. Tooth loss events were recorded for subjects during follow-up. Chi-squared tests were used to study the association between tooth loss and the selected risk factors. Logistic, poisson, and negative binomial regressions were developed to study tooth loss patterns. Overall, $27 \%$ of the subjects lost at least one tooth during follow-up. Fourteen subjects had tooth loss events per 100 person-years. Tooth loss pattern did not differ significantly among different special-needs subgroups (i.e. community-dwelling vs. long-term care, physically disabled $v$ s. functionally independent). Special-needs subjects with three or more active dental conditions at arrival had more than twice the risk of losing teeth than those without any existing conditions. After adjusting other factors, the number of carious teeth or retained roots at arrival was a significant predictor of tooth loss for older adults with special needs $(P=0.001)$. These findings indicate that appropriately managing active caries and associated conditions is important to prevent tooth loss for older adults with special needs.

Keywords: tooth loss; elderly; special needs

International Journal of Oral Science (2011) 3: 27-33. doi: 10.4248/IJOS11012

\section{Introduction}

"Older adults with special needs" refer to medically compromised, physically disabled, or cognitively impaired elders, and those for whom oral health and oral health care are complicated by financial, and/or access factors. Due to loss of cognitive skills, physical disability, self-neglect, and lack of social support and resources for regular dental care, special-needs elders experience more oral disease and conditions compared to healthy, independent elders [1-3]. Compared to age- and gendermatched elders, demented patients may have poorer oral hygiene, and experience a high incidence of caries,

\section{*Correspondence: Xi Chen}

Tel: 919966 8522; Fax: 9198431170

E-mail: xi_chen@dentistry.unc.edu

Received 21 September 2010; Accepted 18 November 2010 periodontal disease, soft tissue pathology, and denturerelated problems [1-8]. Research shows that oral health is poor in long-term care (LTC) residents. Coronal and root caries are highly prevalent in LTC residents, especially those with severe cognitive impairment [9]. An Australian study found that $72.1 \%$ of nursing home (NH) residents developed new coronal and/or root caries in a one-year follow-up period. The coronal and root caries increments among NH subjects were many times greater than that of community-dwelling older adults [10]. Dental plaque accumulation and prevalence of gingivitis are increased among institutionalized elderly $[9,11]$. These findings indicate that older adults with special needs have an increased risk of dental caries and periodontal disease, the common cause of tooth loss.

Given the differences in oral disease patterns, general health, cognitive and physical function, and socioeconomics, the pattern of tooth loss in special-needs elders 
may differ from that in general elderly population. The limited evidences also shows that demented elders tend to have a slightly increased risk of tooth loss compared to non-demented elders $[3,5]$. Although the difference is not statistically significant, demented patients lost on average 1.2 teeth in 5 years, slightly more than 1.0 tooth in non-demented elders [12]. Similar findings can also be found in LTC residents. Tramini and colleagues found that among 321 long-term care residents, more than 50\% had lost 21 or more teeth at the time of examination [13]. A recent study also found that among $260 \mathrm{NH}$ residents, the mean number of functioning teeth was only 10.6 , indicating remarkable oral function loss in these individuals [11]. Another national survey also showed nearly $50 \%$ of nursing home residents had lost their natural teeth completely, and more than $40 \%$ of the residents reported difficulty biting or chewing [14], remarkably higher than the national average edentulous rate in people aged 65 and above [15]. These evidences suggest that tooth loss is a serious issue in special-needs elders. However, most of the evidence has been based on cross sectional studies [11, 13-14, 16-17]. Therefore, the detailed tooth loss pattern in older adults with special needs remains unclear. Although multiple factors, such as old age $[13-14,16,18]$, sociodemographic characteristics [18], poor oral health condition [13, 18], cognitive impairment [17], physical disability [14, 18-19], systemic health and so on $[13,17]$, are considered to be associated with tooth loss in special-needs individuals, how these risk factors interactively and synergistically affect tooth loss in these individuals remains unknown. The lack of understanding on these issues not only increases difficulty for dental professionals in managing tooth loss and associated oral dysfunction for older adults with special needs, but also affects quality of care for these vulnerable individuals.

To address this issue, we conducted a longitudinal study to detail the pattern of tooth loss in a Minnesota elderly cohort with special-needs.

\section{Materials and Methods}

A detailed description regarding study subjects, sample selection and data collection has been reported previously [18]. To facilitate discussion, the materials and methods are briefly described below.

This study was a retrospective longitudinal study approved by University of North Carolina Institutional Review Boards (Study \#: 10-0406), ranging from 10/ 1999 to 12/2006. During the study period, 1626 older adults, including both community-dwelling elderly (38\%) and LTC residents $(62 \%)$, presented as new patients at the study site, a community-based geriatric dental clinic in Minnesota, USA. Among of them, 491 met the selection criteria, 1) presented as a new patient during the observation period and 2) remained dentate after finishing their initial treatment plans and returned for care at least once thereafter, and were retrospectively selected as study subjects. Subjects were treated and brought to a state of oral health before enrollment. The follow-up started when the first treatment plan was completed. Comprehensive dental care was continually provided to the subjects during follow-up.

The study data was abstracted from two sources: dental records and the dental office management system used in the study clinic, and classified into four categories: 1) demographic and socioeconomic factors; 2) oral assessment at arrival; 3) medical assessment at arrival; 4) and functional and cognitive assessment at arrival. Given that there were multiple dental providers involved in caring for the subjects during the study period and calibration between examiners was impossible to be implemented, potential variations might be present in recording the existing conditions (i.e. extensive caries $v s$. retained root). To overcome this limitation, the existing conditions on dental hard tissue were verified by the radiographs of the subjects and grouped together using one variable, number of teeth being carious or retained roots. Similarly, number of teeth with restorations was used to record all the teeth with any type of restorations, including amalgam restoration, composite restoration, gold inlay/onlay, metal or porcelain-fusedto-metal crown, and so on.

The study sample was a dynamic population and the subjects entered the study and dropped out at different points of time during the study period. Therefore, personyear was used as an offset to standardize the data for calculating frequency and rate of tooth loss.

Student's $t$-test, Chi-squared test and Analysis of Variance (ANOVA) were used to study the association between tooth loss and the selected risk factors. An unadjusted relative risk along with a corresponding 95\% confidence interval was calculated for the categories within each risk factor. A poisson regression and a negative-binomial regression were adapted to estimate the incident rate of tooth loss and number of teeth lost per person-year in this special-needs population. A logistic regression was developed to study probability of tooth loss. A candidate multivariate regression was first developed using all the selected variables. Variables with high $P$-values $(P>0.05)$ were then removed using a backward stepwise technique, with each variable's $P$-value reassessed at each step. The final reduced model included only variables that were significant at the 0.05 level. 
SAS 9.2 was used for the data analysis.

\section{Results}

\section{Pattern of tooth loss}

Overall, 134 (27\%) subjects lost 328 teeth during the follow-up, which accounted for $3.7 \%$ of the total teeth presented at the beginning of follow-up. The proportion of subjects losing one or more teeth was slightly higher in LTC residents, but the difference was non-significant $(P=0.86$, Table 1$)$. The overall incident rate of tooth loss in this special-needs population was 14 cases per 100 person years with no difference among different specialneeds subgroups (i.e. community-dwelling vs. long-term care, physically disabled $v s$. functionally independent, data not shown). On average, a special-needs subject lost about one tooth per five person years, with disabled subjects losing slightly more teeth than functionally independent subjects (1.3 vs. 0.8, Rate ratio $=1.7,95 \%$ $\mathrm{CI}=1.1,2.5)$.

More than seventy percent of the subjects with tooth loss events during the follow-up lost one or two teeth (Table 2). About twelve percent of the subjects lost 3 or 4 teeth and more than $14 \%$ lost 5-9 teeth. The incidence of edentulism was low. Overall, only $0.6 \%$ of subjects ( $2.2 \%$ among those with tooth loss) became edentulous during the follow-up. The subjects with physical limitations tended to lose more teeth than those functionally independent subjects, but the difference was non-significant $(P=0.70$, Table 2$)$. Similarly, there was no difference in number of teeth lost between community-dwelling and LTC subjects (data not shown).

Table 1 Percent of subjects losing one or more teeth by residency

\begin{tabular}{lccc}
\hline \multicolumn{1}{c}{ Residency } & $\begin{array}{c}\text { Percent of subjects losing one } \\
\text { or more teeth }\end{array}$ & Relative risk \& 95\% CI & $P$-value** \\
\hline Community subjects $(n=256)$ & 27.0 & Ref* $^{*}$ & 0.86 \\
LTC subjects* $(n=235)$ & 27.7 & $1.03(0.77,1.37)$ & \\
\hline
\end{tabular}

*LTC: Long-term care, including nursing homes and assisted programs. **: Chi-square test.

Table 2 Number of teeth lost by physical limitation

\begin{tabular}{|c|c|c|c|c|c|}
\hline \multirow[b]{2}{*}{ Number of teeth lost } & \multirow[b]{2}{*}{$\begin{array}{c}\text { All subjects }(\%) \\
\quad(n=487)\end{array}$} & \multirow{2}{*}{$\begin{array}{l}\text { Subjects losing one or } \\
\text { more teeth }(\%) \\
(n=134)\end{array}$} & \multicolumn{2}{|c|}{ Physical limitation* } & \multirow{2}{*}{$\begin{array}{c}P \text {-value** } \\
(\text { Yes } v s . \text { No) }\end{array}$} \\
\hline & & & $\begin{array}{c}\text { Yes } \\
(n=266)\end{array}$ & $\begin{array}{c}\text { No } \\
(n=221)\end{array}$ & \\
\hline 0 & 72.9 & - & 74.8 & 70.0 & 0.70 \\
\hline 1 & 15.1 & 55.2 & 14.3 & 16.4 & \\
\hline 2 & 4.7 & 17.2 & 4.5 & 5.0 & \\
\hline $3-4$ & 3.3 & 11.9 & 3.0 & 3.6 & \\
\hline $5-9$ & 3.9 & 14.2 & 3.0 & 5.0 & \\
\hline $10+$ & 0.1 & 1.5 & 0.4 & 0.0 & \\
\hline
\end{tabular}

*: measured by transferability, an important component of activities of daily living (ADL). Subjects walk and transfer themselves into dental chair independently was considered having no physical limitation. **: Chi-square test. Due to missing data, some subjects were excluded from data analysis. Therefore, $n$ may vary from the total number of subjects in difference analysis.

\section{Type of teeth lost}

Overall, maxillary molars had the greatest risk of being lost during the follow-up, followed by mandibular incisors. Compared to other tooth types, canines, especially mandibular canines, were less likely to be lost. Types of teeth lost differed between demented and non-demented subjects. While there was no difference in the lost mandibular teeth between two groups, demented subjects were more likely to lose maxillary posterior teeth $(P=0.04)$. More specifically, maxillary incisors were least likely to be lost in demented subjects, and mandibular incisors were lost most frequently among these subjects. Among non-demented subjects, maxillary incisors were lost most frequently and mandibular canines were least likely to be lost (data not shown).

Factors associated with tooth loss

A bivariate analysis shows that tooth loss was asso- 
ciated with two dental factors, number of teeth at arrival and number of teeth being carious or retained root at arrival. Elderly subjects with moderate to severe cognitive impairment, with physical limitation, or required supervision/help to perform oral hygiene care tended to have increased risk of tooth loss, but the associations were non-significant (data not shown).
Further analysis indicated that dental characteristics at arrival differed in subjects with and without tooth loss during the follow-up (Table 3). On average, subjects losing at least one tooth presented with fewer teeth and more caries/retained roots than those without tooth loss, and a higher proportion of these remaining teeth were either carious or retained roots $(P=0.002)$.

Table 3 Selected dental characteristics at arrival between groups with and without tooth loss $(n=491)$

\begin{tabular}{|c|c|c|c|}
\hline Characteristics at arrival & $\begin{array}{l}\text { Persons losing no teeth } \\
\text { Mean (SD) }\end{array}$ & $\begin{array}{l}\text { Person losing one or more teeth } \\
\text { Mean (SD) }\end{array}$ & $P$-value* \\
\hline Number of remaining teeth at arrival & $18.31(8.41)$ & $14.47(7.57)$ & $<0.001$ \\
\hline Number of teeth being carious or retained roots at arrival & $2.99(4.00)$ & $4.26(3.88)$ & 0.002 \\
\hline Number of teeth with restorations at arrival & $11.34(6.26)$ & $10.65(5.73)$ & 0.27 \\
\hline Percent of teeth being carious or retained roots at arrival / \% & $18.48(24.57)$ & $26.37(24.56)$ & 0.002 \\
\hline Percent of teeth with restorations at arrival $/ \%$ & $57.13(23.57)$ & $57.40(23.33)$ & 0.91 \\
\hline
\end{tabular}

*: Student's $t$-test.

Table 4 Factors associated with tooth loss in older adults with special needs

\begin{tabular}{llcccc}
\hline & & $\begin{array}{c}\text { \% Losing one or } \\
\text { more teeth }\end{array}$ & $\begin{array}{c}\text { Relative risk \& } \\
95 \% \text { CI }\end{array}$ & $\begin{array}{c}P \text {-value* } \\
P \text {-value** }\end{array}$ & $\begin{array}{c}\text { Mean number of } \\
\text { teeth lost } \\
\text { Mean (SD) }\end{array}$ \\
\hline Number of teeth & $1-6(n=39)$ & 18.0 & 1.00 & 0.01 & $0.33(0.93)$ \\
at arrival & $7-12(n=71)$ & 31.0 & $1.73(0.81,3.67)$ & & $1.00(2.19)$ \\
& $13-18(n=87)$ & 39.1 & $2.18(1.06,4.48)$ & $0.98(1.84)$ \\
& $19-24(n=144)$ & 29.2 & $1.63(0.79,3.33)$ & $0.63(1.68)$ \\
Number of teeth & $0(n=146)$ & 19.3 & $1.08(0.51,2.27)$ & $0.46(1.35)$ \\
being carious or & $1-2(n=130)$ & 21.6 & 1.00 & $0.36(1.08)$ \\
retaining roots at & $3-4(n=76)$ & 20.2 & $1.30(0.81,2.11)$ & 0.002 & $0.54(1.45)$ \\
arrival & $\geq 5(n=139)$ & 39.6 & $2.08(1.30,3.31)$ & & $0.84(1.71)$ \\
\hline
\end{tabular}

*: Chi-square test. **: ANOVA.

Compared to those with only a few teeth remaining at arrival, subjects presenting with more teeth tended to have an increased risk to losing teeth during the followup, especial those with $13-18$ teeth at arrival $(R R=2.2$, 95\% CI 1.06-4.48). The number of teeth lost also followed a similar pattern. The mean number of teeth lost in subjects with 1-6 teeth at arrival was 0.33 , significantly lower than 1.0 and 0.98 in those with 7-12 and 13-18 teeth at arrival, respectively (Table 4). The number of teeth being carious or retained root at arrival also played an important role in the risk of tooth loss in this population. Subjects presenting with three or more existing dental conditions had more than twice the risk of losing at least one tooth compared to those without any existing condition on dental hard tissue. The mean number of teeth lost in subjects with five or more teeth being carious or retaining roots at arrival was about three times that in those without any caries or related dental conditions $(P=0.005$, Table 4$)$.

\section{Multivariate analysis}

A multivariate logistic regression was developed to identify predictors of tooth loss. After adjusting other factors, number of teeth being carious or retained roots at arrival was the only risk factor significantly associated with tooth loss $(P=0.001$, Table 5). Holding all other 
Table 5 The logistic regression model to predict risk of tooth loss

\begin{tabular}{lcccc}
\hline \multicolumn{1}{c}{ Covariate } & Parameter estimate & Standard error & $P$-value & Odds ratio and 95\% CI \\
\hline $\begin{array}{l}\text { Intercept } \\
\begin{array}{l}\text { Number of teeth being carious or retaining } \\
\text { roots at arrival }\end{array}\end{array}$ & -1.28 & 0.15 & $<0.0001$ & - \\
\hline
\end{tabular}

predictors constant, a one unit increase in the number of teeth being carious or retained roots at arrival corresponds to an increased likelihood of $1.1(95 \% \mathrm{CI}=1.03,1.14)$ for tooth loss.

\section{Discussion}

This study detailed patterns of tooth loss for older adults with special needs. Among 491 subjects, 27\% of the subjects lost at least one tooth during the follow-up (mean=39). The incident rate of tooth loss was 14 cases per 100 person-years. On average, special-needs subjects lost about one tooth every five person-years, with disabled subjects losing slightly more teeth per personyear than non-disable subjects. Tooth loss pattern did not differ significantly among different special-needs subgroups (i.e. community-dwelling vs. LTC, physically disabled $v s$. functionally independent). Tooth loss was significantly associated with dental caries and related conditions. A one unit increase in number of teeth being carious or retaining roots at arrival means the likelihood of tooth loss will increase by 1.1 times. These results provide useful information for dental professionals to appropriately prevent and manage tooth loss for specialneeds elders.

The subjects were treated and brought to a state of oral health before enrollment. The follow-up started when the first treatment plan was completed. At this point, subjects were considered disease-free and their oral function was deemed relatively stable. The selection of the study's time origin was appropriate given the unique characteristics of the study population. The study clinic was a not-for-profit geriatric dental clinic. A great majority of the patients who received care in this clinic were low-income elderly and those with special-needs. Due to lack of resources for dental care, physical/ mental disability, lack of social support and transportation, neglect and other reasons, special-needs elders experience more difficulties in accessing necessary dental care than their healthy, independent counterparts [20-24]. Consequently, these individuals usually presented to the study clinic with poor oral health, including multiple infected or unrestorable teeth. In our opinion, these infected or unrestorable teeth are the cumulative results of different oral diseases and lack of access to regular dental care for long periods of time. Therefore, including the teeth that were extracted during the initial treatment into the analysis would inflate the tooth loss rate and might not truly reflect the risk of tooth loss in this population, especially for those who had not had dental care for a long-period time (i.e. those who intentionally held off dental care until being eligible for Minnesota Medicaid program).

Out of 487 subjects, $15.1 \%$ of the subjects lost one tooth during the follow-up; $4.7 \%$ lost two teeth, 3.3\% lost three to four teeth and $3.9 \%$ lost five or more teeth. This pattern is similar with that in the Florida Dental Care Study [25]. However, given the fact that all subjects were disease-free at enrollment and routine dental care was continually provided to subjects during follow-up, the severity of tooth loss in this special-needs cohort may be more considerable than those reported in the previous studies [25-26] in which many study subjects might enroll with active dental diseases and conditions. Also, although more than one-fourth of the subjects had tooth loss events during the follow-up, only $3.7 \%$ of the teeth presented at the beginning of the follow-up were lost. Among them, maxillary molars were most likely to be lost, followed by mandibular incisors. Compared with other types of teeth, mandibular canines were least likely to be lost. A similar pattern has been reported in the studies in Iowa [27] and China [28].

A bivariate analysis found that, except for those with only 1-6 teeth remaining, subjects presenting with fewer teeth at arrival had a higher risk of losing teeth during the follow-up, especially those with 13-18 teeth at arrival. These findings, together with the results of previous studies [26-27], indicate that past history of tooth loss is an important predictor for future tooth loss in this special-needs cohort. Compared with other groups, subjects with 1-6 remaining teeth had a lower risk to lose teeth. This may be associated with the current model of dental care in which tooth loss not only results from clinical pathology, but is also associated with the treatment planning judgments of the dental professionals involved in care [29], and the preferences, values and available resources for dental care of patients or their responsible parties $[27,29]$. Loss of one tooth could potentially have a significant impact on the oral function of those with only 1-6 teeth remaining at enrollment, depending on 
the location of the tooth in the dentition and if this tooth is supporting a dental prosthesis. Under this circumstance, patients and/or their responsible parties might be hesitant to have any teeth extracted. The dentists involved in care might also be more conservative during treatment planning and be reluctant to extract the remaining teeth. As a result, risk of tooth loss was lowered in these patients.

Number of teeth being carious or retained roots at arrival, an indicator of existing dental conditions, is an important risk factor associated with tooth loss in special-needs elders. Subjects who lost at least one tooth not only presented with more caries or retained roots, but the proportion of the remaining teeth being carious or retained roots was also higher. Compared to those without active dental conditions at arrival, subjects who presented with three or more teeth being carious or retained roots had more than twice the risk to lose teeth during follow-up. Multivariate logistic analysis also revealed that number of teeth being carious or retained roots was the only risk factor associated with subsequent tooth loss in this cohort. This result corroborates the findings of the previous studies [25-26, 28, 30] and indicates that dental caries and/or its associated conditions is an important predictor for tooth loss, not only in general elderly population but also in older adults with special needs. Although a one unit increase in number of teeth being carious or retained roots at arrival only results in a 1.1 times increase in risk of subsequent tooth loss, which seems clinically insignificant, given that nearly $30 \%$ of the subjects presented with 5 or more decayed/broken teeth, risk for subsequent tooth loss will increase at least 1.6 times in these individuals. Therefore, for elderly patients presenting with multiple existing dental caries, thorough risk assessment and adequate preventive care plans need to be established to prevent tooth loss. This is particularly important for those with cognitive and/or functional impairment.

Previous studies found that physical disability is associated with tooth loss in special-needs individuals $[14,19,31]$. However, functional assessment based on standard instruments, such as activity of daily living (ADL), were not available in the dental records. We were therefore unable to assess the impact of physical function impairment on tooth survival in the elderly subjects using the standard functional assessment instruments. Given that more than $40 \%$ of the subjects presented with some sort of physical limitation, we used transferability, an important component of ADL, together with capacity to perform oral hygiene care to assess the impact of functional impairment on tooth loss. We found no significant association between tooth loss and these two variables. However, given these variables cannot fully represent physical functional status of study subjects, further efforts are necessary to better understand the association between physical function impairment and tooth loss. Cognitive impairment is also associated with increased tooth loss in dementia patients [9, 31-32]. However, since cognitive assessment based on standard instruments (e.g. Mini Mental Status Examination) is not widely available in clinical dental settings, including in the study clinic, we were unable to precisely measure the association between cognitive impairment and tooth loss in this study. Moreover, potentially important risk factors, including periodontal health, salivary flow, use of preventive care, and baseline oral hygiene status, were either not available or not available electronically and were not included in the analysis. Finally, this study was conducted based on a community-based geriatric dental clinic in which a large proportion of the subjects had special-needs and dental coverage through the Medicaid program. This study sample could have different characteristics and disease patterns when compared to the general elderly population [33]. Additionally, the specific practice pattern and treatment philosophy employed in this clinical setting might also differ from other general dental practices, which therefore affects tooth survival in the study population. While the findings of this study may be applicable to the elderly patients who are older, medically compromised or with special-needs, further work is needed to generalize the results of this study to the general elderly population.

\section{Conclusion}

Tooth loss did not differ significantly among different special-needs subgroups (i.e. community-dwelling vs. LTC, physical disable $v s$. functionally independent). The incident rate of tooth loss in older adults with special needs was fourteen cases per 100 person-years. The elderly subject lost one tooth per 5 person years. The special-needs subjects with three or more active dental conditions had more than twice the risk to lose teeth compared to those without any existing condition at arrival. After adjusting other factors, the number of active dental caries and associated conditions is an important risk factor to predict tooth loss. These findings indicate that appropriate management of dental caries is important to prevent tooth and oral function loss in older adults with special needs.

\section{References}

1 Berg R, Berkey DB, Tang JM, Baine C, Altman DS. Oral health status of older adults in Arizona: results from the 
Arizona elder study. Spec Care Dentist 2000; 20: 226-233.

2 Chalmers JM, Carter KD, Spencer AJ. Caries incidence and increments in community-living older adults with and without dementia. Gerodontology 2002; 19: 73-88.

3 Chalmers JM, Carter KD, Spencer AJ. Oral diseases and conditions in community-living older adults with and without dementia. Spec Care Dentist 2003; 23: 7-17.

4 Jones JA, Lavellee N, Alman J, Sinclair C, Garcia RL. Caries incidence in patients with dementia. Gerodontics 1993; 10: 76-82.

5 Ship JA, Puckett SA. Longitudinal study on oral health in subjects with Alzheimer's disease. JAGS 1994; 42: 57-63.

6 Warren JJ, Chalmers JM, Levy SM, et al. Oral health of persons with and without dementia attending a geriatric clinic. Spec Care Dentist 1997; 17: 47-53.

7 Ghezzi EM, Ship JA. Dementia and oral health. Oral Surg Oral Med Oral Pathol 2000; 89: 2-5.

8 Henriksen BM, Engedal K, Axéll T. Cognitive impairment is associated with poor oral health in individuals in long-term care. Oral Health Prev Dentist 2005; 3: 203-207.

9 Chalmers JM, Hodge C, Fuss JM, Spencer AJ, Carter KD. The prevalence and experience of oral diseases in Adelaide nursing home residents. Aust Dent J 2002; 47: 123-130 .

10 Chalmers JM, Carter KD, Spencer AJ. Caries incidence and increments in Adelaide nursing home residents. Spec Care Dentist 2005; 25: 96-105.

11 Peltola P, Vehkalahti MM, Wuolijoki-Saaristo K. Oral health and treatment needs of the long-term hospitalised elderly. Gerodontology 2004; 21: 93-99.

12 Chen X, Shuman SK, Hodges JS, Gatewood LC, Xu J. Patterns of tooth loss in older adults with and without dementia: a retrospective study based on a Minnesota cohort. $J A G S$ (In press).

13 Tramini P, Montal S, Valcarcel J. Tooth loss and associated factors in long-term institutionalized elderly patients. Gerodontology 2007; 24: 196-203.

14 Gift HC, Cherry-Peppers G, Oldakowski RJ. Oral health status and related behaviors of U.S. nursing home residents, 1995. Gerodontology 1998; 14: 89-99.

15 The Centers for Disease Control and Prevention (CDC). Trends in oral health status: United States, 1988-1994 and 1999-2004. Series 11, Number 248. 104 pp. (PHS) 20071698. on World Wide Web http://www.cdc.gov/nchs/data/ series/ sr_11/sr11_248.pdf. [accessed on 12/7/2009].

16 Murray PE, Ede-Nichols D, Garcia-Godoy F. Oral health in Florida nursing homes. Int J Dent Hyg 2006; 4: 198-203.

17 Wu B, Plassman BL, Crout RJ, Liang J. Cognitive function and oral health among community-dwelling older adults. $J$ Gerontol A Biol Sci Med Sci 2008; 63: 495-500.

18 Chen X, Hodges JS, Shuman SK, Gatewood LC, Xu J.
Predicting tooth loss for older adults with special needs. Community Dent Oral Epidemiol 2010; 38: 235-243.

19 Holm-Pedersen P, Schultz-Larsen K, Christiansen N, Avlund $\mathrm{K}$. Tooth loss and subsequent disability and mortality in old age. J Am Geriatr Soc 2008; 56: 429-435.

20 Weiss RT, Morrison BJ, MacEntee MI, Waxler-Morrison NE. The influence of social, economic, and professional considerations on services offered by dentists to long-term care residents. JPHD 1993; 53: 70-75.

21 Weeks JC, Fiske J. Oral care of people with disability: a qualitative exploration of the views of nursing staff. Gerodontology 1994; 11: 13-17.

22 Johnson TE, Lange BM. Preferences for and influences on oral health prevention: perceptions of directors of nursing. Spec Care Dentist 1999; 19: 173-180.

23 Chalmers JM, Hodge C, Fuss J, et al. Opinions of dentists and directors of nursing concerning dental care provision for Adelaide nursing homes. Aust Dent J 2001; 46: 277-283.

24 Chalmers JM, Pearson A. A systematic review of oral health assessment by nurses and carers for residents with dementia in residential care facilities. Spec Care Dentist 2005; 25: 227-233.

25 Gilbert GH, Miller MK, Duncan RP, et al. Tooth-specific and person-level predictors of 24-month tooth loss among older adults. Community Dent Oral Epidemiol 1999; 27: 372-385.

26 Locker D, Ford J, Leake JL. Incidence of and risk factors for tooth loss in a population of older Canadians. J Dent Res 1996; 75: 783-789.

27 Hand JS, Hunt RJ, Kohout FJ. Five-year incidence of tooth loss in Iowans aged 65 and older. Community Dent Oral Epidemiol 1991; 19: 48-51.

28 Baelum V, Luan WM, Chen X, Fejerskov O. Predictors of tooth loss over 10 years in adult and elderly Chinese. Community Dent Oral Epidemiol 1997; 25: 204-210.

29 Johnson TE. Factors contributing to dentists' extraction decision in older adults. Spec Care Dentist 1993; 13: 195 199.

30 Slade GD, Gansky SA, Spencer AJ. Two-year incidence of tooth loss among South Australians aged 60+ years. Community Dent Oral Epidemiol 1997; 25: 429-437.

31 Chen X. Development of a prognostic index to predict tooth survival for older adults ( $\mathrm{PhD}$ dissertation). Minneapolis, MN. Univ. of Minnesota, 2008.

32 Rejnefelt I, Andersson P, Renvert S. Oral health status in individuals with dementia living in special facilities. Int $J$ Dent Hyg 2006; 4: 67-71.

33 Administration on Aging, U.S. Department of Health and Human Services. A profile of older Americans: 2009. on World Wide Web http://www.aoa.gov/aoaroot/aging_satistics/profile/ index.aspx. [accessed on 4/17/2010]. 